\title{
Considerações sobre a síndrome da disfunção autonômica pós- traumatismo cranioencefálico: fisiopatologia e tratamento
}

\author{
Francisco Neuton Magalhães ${ }^{1}$, Wellingson Silva Paiva ${ }^{1}$, Almir Ferreira \\ de Andrade ${ }^{2}$, Edson Bor-Seng-Shu ${ }^{3}$, Rodrigo Moreira Faleiro, \\ Eberval Gadelha Figueiredo ${ }^{5}$, Manoel Jacobsen Teixeira ${ }^{6}$
}

Divisão de Neurocirurgia do Hospital das Clínicas da Faculdade de Medicina da Universidade de São Paulo, SP; Unidade de Neurocirurgia do Hospital João XXIII, Belo Horizonte, MG.

\section{RESUMO}

O termo síndrome da disautonomia pós-traumática é usado para identificar uma síndrome de hiperatividade simpática e muscular paroxística após TCE. Essa síndrome representa uma afecção grave, frequentemente subestimada na assistência ao paciente com neurotrauma e que resulta em piora da recuperação funcional e elevação dos custos hospitalares por aumento do tempo de internação. Neste artigo, realizamos uma revisão crítica da literatura sobre os princípios fisiopatológicos e abordagem terapêutica na síndrome da disautonomia no paciente com traumatismo cranioencefálico.

\section{PALAVRAS-CHAVE}

Trauma, traumatismos encefálicos/fisiopatologia, sistema nervoso autônomo, disautonomias primárias, revisão.

\section{ABSTRACT}

Dysautonomia after traumatic brain injury: physiopathology and treatment

Dysautonomic post-traumatic syndrome consists in a paroxystic condition sympathetic and muscle hyperactivity after TBI. This syndrome is a serious condition, often underestimated in assisting the patient with neurotrauma and that result in worsening of functional recovery and increased hospital costs by increasing the length of stay. In this paper, we conducted a critical review of the literature on the physiopathological and therapeutic mechanisms in the syndrome of autonomic dysfunction in patients with traumatic brain injury.

\section{KEYWORDS}

Trauma, brain injury/physiopathology, autonomic nervous system, dysautonomia, review.

1 Médico-assistente da Divisão de Neurocirurgia do Hospital das Clínicas da Faculdade de Medicina da Universidade de São Paulo (HC-FMUSP), São Paulo, SP, Brasil.

2 Coordenador da Unidade de Emergência da Divisão de Neurocirurgia do HC-FMUSP, São Paulo, SP, Brasil.

3 Coordenador da Unidade de Neurossonologia da Divisão de Neurocirurgia do HC-FMUSP, São Paulo, SP, Brasil.

4 Médico-assistente da Unidade de Neurocirurgia do Hospital João XXIII, Belo Horizonte, MG, Brasil.

5 Coordenador do Grupo de Doenças Cerebrovasculares; supervisor da Divisão de Neurocirurgia do HC-FMUSP, São Paulo, SP, Brasil.

6 Professor titular da Divisão de Neurocirurgia do HC-FMUSP, São Paulo, SP, Brasil. 


\section{Introdução}

O traumatismo cranioencefálico (TCE) é a principal causa de morte e sequela em crianças e adultos jovens nos países industrializados ocidentais. ${ }^{1,2}$ Nos Estados Unidos, estima-se em torno de 500 mil novos pacientes com TCE por ano. Desses, cerca de 50 mil morrem antes de chegar ao hospital e mais 15 a 20 mil falecem após atendimento hospitalar. Dos cerca de 430 mil restantes, outros 50 mil evoluirão com sequelas neurológicas de maior ou menor gravidade. ${ }^{3}$ Embora a causa principal do TCE varie entre diferentes localidades, os acidentes de trânsito, as quedas e as agressões estão entre suas causas mais frequentes. ${ }^{4} \mathrm{~A}$ taxa de mortalidade também sofre grande variação, sendo mais baixa em países com menor violência no trânsito. ${ }^{5}$ Entre o grande número de sobreviventes, uma parcela importante desenvolve complicações neurológicas e sistêmicas, dentre as quais se destacam os quadros de disautonomia.

A ativação do sistema nervoso autônomo é uma consequência normal da resposta metabólica ao trauma. ${ }^{6}$ No período pós-traumático imediato, achados comuns incluem taquicardia, aumento da pressão sanguínea e redirecionamento do fluxo sanguíneo para os músculos e para o cérebro. Essas mudanças hemodinâmicas têm o objetivo de assegurar a disponibilidade de oxigênio e glicose para o cérebro e manter outros processos fisiológicos vitais. Após sobreviver à lesão inicial, o organismo pode esperar uma ativação simpática aumentada em resposta a novos desafios, tais como infecção, dor e retirada de drogas. ${ }^{6-8}$

Em muitas situações, o grau da resposta autonômica é um indicador da gravidade do processo patológico. Entretanto, como em qualquer processo fisiológico, uma resposta exacerbada tem o potencial de desviar da proteção para a agressão, podendo causar morbidade adicional. Essas situações ocorrem após trauma do sistema nervoso central [traumatismo cranioencefálico e trauma raquimedular (TRM)]. Entre essas duas situações, as consequências autonômicas são mais compreendidas no TRM que no TCE. ${ }^{6}$

Os distúrbios autonômicos em paciente com TCE constituem uma condição clínica grave e frequentemente pouco valorizada pelas equipes médicas. $\mathrm{O}$ termo disautonomia é usado para identificar uma síndrome de hiperatividade simpática e muscular paroxística pós-TCE. ${ }^{9}$ As mudanças autonômicas incluem significante aumento da frequência cardíaca (FC) e da frequência respiratória (FR), elevação da temperatura, aumento da pressão arterial (PA) sistêmica e sudorese; as alterações musculares consistem em postura de descerebração ou decorticação, distonia, rigidez e espasticidade. Grande parte da literatura publicada é proveniente de estudos de casos ou pequenas séries de pacientes que sofreram
TCE; entretanto, essa síndrome tem sido relatada em uma grande variedade de lesões neurológicas, tais como acidente vascular encefálico, hipóxia cerebral na ausência de outras lesões, hidrocefalia aguda, tumores cerebrais, aumentos da pressão intracraniana e, em particular, hemorragia subaracnóidea espontânea ou intraparenquimatosa. ${ }^{10}$ Por causa dessas alterações, acredita-se que o estado de hipertermia prolongada, o catabolismo excessivo, os altos níveis séricos de catecolaminas e a espasticidade/distonia aumentem significativamente a morbimortalidade. Atualmente, existem incertezas acerca dos mecanismos fisiopatológicos envolvidos, os quais serão abordados nas seções seguintes. ${ }^{6}$

Estados de hiperatividade simpática paroxística e alterações motoras também podem ocorrer em outros distúrbios relacionados a neurotransmissores. ${ }^{6}$ Essas "síndromes de sobreposição" incluem síndrome neuroléptica maligna, síndrome serotoninérgica, retirada aguda de baclofeno intratecal, síndrome hiperpirética parkinsoniana, catatonia maligna, hipertermia maligna e síndrome do homem rígido. Além disso, sintomas semelhantes podem resultar da exposição a certos venenos animais, produtos químicos e agentes infecciosos, como o tétano e a raiva. Uma variante de curta duração parece acontecer em alguns pacientes que recebem eletroconvulsioterapia.?

\section{Nomenclatura}

A primeira descrição dessa síndrome foi publicada por Penfield em 1929 com o nome de "crises autonômicas diencefálicas". ${ }^{11}$ Entretanto, ao longo dos anos, vários termos têm sido empregados para definir essa entidade clínica, tais como "tempestade cerebral", "desregulação central", "reação autonômica de emergência", "estado hiperadrenérgico", "síndrome aguda do tronco cerebral", "disautonomia", "resposta simpático-adrenal", "tempestade simpática e autonômica", "hiperatividade simpática paroxística, entre outros. ${ }^{8}$ Atualmente, ainda não existe um termo de consenso na literatura, mas, em função da compreensão fisiopatológica dessa condição, o termo mais empregado é "síndrome da disfunção autonômica" ou "disautonomia".-10

\section{Incidência}

As melhores estimativas da incidência dessa síndrome derivam de estudos de neurointensivismo com 
pacientes que sofreram TCE grave, com valores que variam de $8 \%$ a $33 \%{ }^{6,8,9}$

Em um estudo prospectivo recente com 101 pacientes com TCE grave, Lv et al. ${ }^{10}$ descrevem uma incidência de 20,6\% dos pacientes com síndrome de disautonomia pós-TCE e relatam, ainda, que sexo do paciente, tipo de lesão, história de hipertensão, pressão arterial sistólica de admissão, presença de fratura, lesão pulmonar, escore de gravidade de trauma, sedação ou analgesia e craniotomia de emergência não influenciaram na incidência da disautonomia. Nesse mesmo estudo um modelo de regressão logística multivariada revelou que idade do paciente e DAI foram dois preditores independentes de disautonomia.

\section{Manifestações clínicas}

O curso clínico da síndrome da disautonomia pós-TCE (SDA) parece ser composto de três fases. No período pós-traumático inicial (primeira fase), é quase impossível distinguir os pacientes que irão desenvolvê-la; na segunda fase, que começa geralmente com a retirada da sedação, os primeiros sinais clínicos aparecem nos pacientes acometidos. Na primeira parte dessa segunda fase, os episódios são frequentes, prolongados e intensos. À medida que o tempo passa, os sinais de paroxismo simpático tornam-se menos pronunciados na duração, frequência e magnitude. O padrão de resposta motora é frequentemente assimétrico e variável, não necessariamente assumindo posturas de descerebração ou decorticação. O padrão da sudorese também se altera, deixando de ser no corpo inteiro para se concentrar na parte superior do tronco, cabeça e pescoço, antes de cessar completamente. ${ }^{12} \mathrm{O}$ desaparecimento da sudorese é usado como um marco na transição entre a segunda e a terceira fase, ocorrendo aproximadamente no $74^{\circ}$ dia após a lesão inicial., ${ }^{6,13}$

Dentre os pacientes com TCE, aqueles que desenvolveram SDA têm os piores resultados funcionais, anormalidades de deglutição prolongadas, longo período de amnésia pós-traumática, longos períodos de internação e alto custo hospitalar. Embora esses pacientes comecem a se recuperar num status neurológico baixo, tem sido percebido que o final dos períodos de paroxismos coincide com a melhora do status neurológico. Entretanto, muitos pacientes continuam com anormalidades motoras tipo rigidez, distonia e espasticidade. ${ }^{6,14} \mathrm{Em}$ alguns casos, a gravidade da limitação física pode se apresentar como a "síndrome do encarceramento", o que pode limitar uma avaliação cognitiva apropriada. Pacientes com SDA geralmente não apresentam sinais evidentes de hiperatividade autonômica na fase crônica, mas anormalidades nos mecanismos neurológicos de controle da função cardíaca persistem pelo menos por 14 meses após o TCE. Esses pacientes também têm risco elevado de desenvolver ossificação ectópica. ${ }^{6,11}$

\section{Dificuldades no diagnóstico}

Existem várias questões que precisam ser consideradas para se estabelecer o diagnóstico da síndrome de disautonomia. Clinicamente, o reconhecimento dessa síndrome requer um alto nível de suspeição e o diagnóstico permanece como de exclusão. ${ }^{6,15}$ Para complicar o contexto, os paroxismos disautonômicos podem coexistir com outras situações clínicas agudas tipo sepse, dor e retirada de drogas, aumentando a probabilidade de que um componente paroxístico subjacente permaneça irreconhecível. Dentre as enfermidades com apresentação paroxística, as crises epilépticas, especialmente do tipo não convulsivo e focais, necessitam exclusão por eletroencefalograma. ${ }^{16}$ Além disso, alguns achados clínicos da SDA podem se sobrepor a outras entidades clínicas originadas a partir do desequilíbrio entre neurotransmissores, como citamos anteriormente. ${ }^{17}$

Além das manifestações autonômicas e dos parâmetros motores, alguns autores têm acrescentado outros sinais clínicos ao diagnóstico. Foram descritos: mudanças na pressão intracraniana (elevação e diminuição); elevação do nível sérico da creatinina-quinase; aumento dos glóbulos brancos na ausência de infecção; arritmias assintomáticas; redução da sudorese e doença pulmonar neurogênica. Outros achados incluem mudanças pupilares, salivação, lágrimas, soluços, choros e suspiros. ${ }^{6,14}$

Alguns autores têm relatado uma associação intermitente entre o início dos paroxismos autonômicos e determinados estímulos. Tais estímulos incluem dor, aspiração endotraqueal, movimentos passivos (torção, estiramento muscular), constipação ou retenção urinária, estímulo emocional e estímulos ambientais do tipo sonoro. ${ }^{13}$

Baguley et al. ${ }^{9}$, em 1999, propôs uma definição de critérios para padronizar pesquisa sobre o tema. Essa definição requer, simultaneamente, aumentos dos paroxismos em pelo menos cinco dos sete achados clínicos comumente relatados (aumentos da frequência cardíaca e respiratória, da temperatura, da pressão arterial sistêmica, sudorese, postura e/ou distonia) em pacientes admitidos para reabilitação. Os parâmetros dessas alterações autonômicas foram baseados nas pesquisas de Jennet e Teasdale na década de 1970, isto é, temperatura acima de $39^{\circ} \mathrm{C}, \mathrm{FC}>120 \mathrm{bpm}, \mathrm{FR}>30$ irpm e PA sistólica $>160 \mathrm{mmHg}$. Em outra pesquisa 
subsequente, os critérios foram alterados discretamente, passando a exigir a presença de paroxismos contínuos por pelo menos 14 dias após a lesão inicial. ${ }^{18} \mathrm{Em} 2004$, Blackman et al. ${ }^{8}$ propuseram um critério similar para diagnosticar a SDA que requer pelo menos um paroxismo por dia em três dias consecutivos nos pacientes vítimas de TCE grave.

Entretanto, ambas as definições possuem falhas. Os valores absolutos dos parâmetros fisiológicos carecem de critérios rígidos definidores e seus valores absolutos não podem ser utilizados apropriadamente para respaldar condutas precoces. Além disso, sabe-se que nem todos os pacientes com SDA desenvolvem todos os sinais clínicos da síndrome e que não é específica de pacientes com TCE grave. Por fim, tem sido proposto que uma hiperatividade a estímulos menores, chamados de "gatilhos" dos paroxismos, poderia ser acrescentada aos critérios diagnósticos, como relatado recentemente num estudo prospectivo. ${ }^{17,18}$

\section{Fisiopatologia}

Existem atualmente duas teorias principais denominadas de epilepsia autonômica e síndromes de desconexão. Dados obtidos de pesquisas clínicas observacionais sugerem que a SDA seria resultado de uma desconexão estrutural ou funcional (tal como aumento da pressão intracraniana ou bloqueio de neurotransmissores), com a tendência de desenvolver paroxismos, sendo mais relacionada a lesões mesencefálicas que diencefálicas. Dessa forma, duas teorias de desconexão foram propostas. A teoria mais aceita, denominada de convencional, sugere que os paroxismos teriam origem em centros excitatórios localizados na porção superior do tronco cerebral ou no diencéfalo. Em contraste, o modelo da razão excitação/inibição advoga que esses centros são naturalmente inibitórios e que os paroxismos seriam mediados por estímulos excitatórios medulares. ${ }^{8}$ Esse modelo engloba os achados clínicos das síndromes de emergência autonômica, acomoda os gatilhos dos paroxismos e explica os efeitos observados após o uso de algumas medicações. ${ }^{9}$

A teoria da disautonomia por desconexão estrutural atribui sua origem a lesões cerebrais com localização e extensão variáveis, partindo do córtex cerebral em direção ao diencéfalo, porção superior do tronco cerebral e medula espinhal, o que resulta na perda da regulação autonômica normal devido à ausência do controle cortical/subcortical. ${ }^{13}$ Isso ocorre nos pacientes com TCE grave que têm frequentemente uma combinação de lesões focais e difusas. Centros excitatórios simpáticos (CES) do hipotálamo e do tronco cerebral seriam os responsáveis pelos paroxismos, uma vez liberados do controle cortical. No entanto, duas dificuldades potenciais com essa teoria precisam ser consideradas. Em primeiro lugar, para que esses CES não identificados pudessem promover os paroxismos, a extensão caudal da lesão deveria ser mínima e esse dano não teria a capacidade de prevenir hiperexcitação. Em segundo lugar, a teoria apresentada ainda não tem modelos testáveis que possam impulsionar pesquisas futuras. ${ }^{9}$

No modelo da desconexão funcional, os paroxismos acontecem em função do processamento anormal (no tronco cerebral/diencéfalo) de estímulos aferentes provenientes da medula espinhal. De acordo com essa teoria, a SDA seria uma das síndromes autonômicas de hiperexcitabilidade, citadas anteriormente. Nessa teoria, a tendência a desenvolver episódios de hiperexcitabilidade é denominada de tendência alodínica. ${ }^{7}$ Por definição, alodínea se refere a um processo de sensitização central que ocorre no corno posterior da medula espinhal, no qual estímulos não dolorosos são percebidos como nociceptivos. Essa tendência alodínica é normalmente controlada por inibição tônica de centros dincefálicos e alguma lesão nesses centros ou distalmente libera o controle desse processo. Uma das vantagens dessa teoria é que existem modelos testáveis por meio de síndromes autonômicas agudas conhecidas. E o mais relevante desse modelo é a proposição de um tratamento eficaz com medicações que diminuem diretamente a tendência alodínica ou aumentam a inibição no tronco cerebral, como a gabapentina. ${ }^{9}$

\section{Tratamento}

As informações sobre os neurotransmissores envolvidos na etiologia da SDA têm sido obtidas a partir de observações clínicas da resposta ao tratamento. Antes de 2004, as melhores evidências para tratar essa síndrome eram com morfina intravenosa, benzodiazepínicos, propranolol, bromocriptina e baclofeno intratecal (BIT). ${ }^{7}$ Mais recentemente, gabapentina também tem se mostrado um agente efetivo. ${ }^{10}$ Enquanto nenhuma dessas medicações for testada em estudos bem controlados, as evidências para sua eficácia são discutidas com base nas teorias que tentam explicar a fisiopatologia dessa síndrome.

A morfina controla a dor e modifica as mudanças extremas no sistema nervoso autônomo e a postura distônica por causa da supressão do fluxo simpático. Em geral, os efeitos são dose-dependentes e alguns pacientes necessitam de doses acima de $20 \mathrm{mg}$ (intravenoso) para eliminar os episódios paroxísticos ${ }^{19}$ (Tabela 1$)$. 
Tabela 1 - Medicações utilizadas no tratamento de disautonomia pós-TCE

\begin{tabular}{llll}
\hline Medicação & Classificação & Uso habitual & Efeitos colaterais \\
\hline Morfina/fentanil & Agonista opioide & Analgesia & Sedação \\
Bromocriptina & Agonista dopaminérgico & Tumores de hipófise/Infertilidade & Convulsão/uso cuidadoso em nefropatas e hepatopatas \\
Carbidopa/levodopa & Agonista dopaminérgico & Doença de Parkinson & Convulsão/uso cuidadoso em nefropatas e hepatopatas \\
Propanolol & Antagonista beta adrenérgico & Hipertensão arterial & Hipotensão/uso cuidadoso em broncopatias \\
& & & e vasculopatias periféricas \\
Clonidina & Agonista alfa-2 adrenérgico & Hipertensão arterial & Hipotensão/Hipertensão rebote \\
Midazolan/diazelan/clonazepam & Agonista GABA A & Sedação & Sedação \\
Baclofeno & Agonista GABA B & Espasticidade & Sedação/bradicardia \\
Clorpromazina & Antagonista dopaminérgico & Hipertermia maligna & Sedação/quadros extrapiramidais \\
Fenitoína/carbamazepina & Anticonvulsivante & Epilepsia & Sedação/Lesões cutâneas \\
Gabapentina & Anticonvulsivante & Epilepsia/dor neuropática & Sedação \\
\hline
\end{tabular}

Tem sido relatado que os agentes dopaminérgicos têm eficácia variável na redução dos paroxismos, podendo cessá-los e diminuir a temperatura e a sudorese. ${ }^{13}$

Existem evidências de que os neurotransmissores simpáticos estão aumentados na SDA. Por isso, tanto alfa-agonistas como betabloqueadores têm sido utilizados no tratamento, sendo clonidina e propranolol os agentes mais estudados. A clonidina, um alfa- 2 agonista, tem ação central e periférica na inibição de centros simpáticos (teoria convencional), ${ }^{17}$ porém os estudos mostram evidências limitadas para sua eficácia na SDA. O propranolol diminui as catecolaminas circulantes e reduz o trabalho cardíaco e o catabolismo. Adicionalmente, o propranolol penetra na barreira hematoencefálica e bloqueia os receptores 5HT1A, sugerindo dois outros mecanismos potenciais para sua eficácia na SDA. ${ }^{13}$

O baclofeno, um agonista GABA B, é administrado no espaço intratecal para pacientes com espasticidade grave quando outras opções de tratamento falharam. O baclofeno intratecal (BIT) atua na inibição de interneurônios na medula espinhal, evitando os efeitos que ocorrem com sua administração por via oral; sabe-se também que a penetração encefálica é mínima. Em nível medular, o BIT teria um duplo efeito antinociceptivo e antiespasticidade. Entretanto, apesar dos efeitos positivos do BIT, sua base anatômica e farmacológica ainda não está completamente compreendida. Além disso, a retirada aguda do BIT pode exacerbar a SDA ou produzir uma SDA em pacientes sem disautonomia. ${ }^{7}$

Recentemente, a gabapentina tem se mostrado mais eficaz que o BIT. Esse fármaco foi desenvolvido inicialmente como anticonvulsivante, mas sua utilização clínica principal é no tratamento de dores neuropáticas por meio de uma ação nas subunidades alfa-2-delta de canais de cálcio voltagem-dependente localizados no corno posterior da medula espinhal., ${ }^{9,20}$ Embora a gabapentina atue tanto no cérebro quanto na medula, seu efeito medular parece assumir maior importância na DAS. Baseado nessa ação, tanto a gabapentina como o BIT atuariam normalizando a modulação dos estímulos aferentes nos pacientes com SDA mediante o aumento da inibição dentro da medula espinhal. A ação desses medicamentos fortalece o conceito do modelo da desconexão funcional na etiologia da SDA. ${ }^{7}$

\section{Considerações finais}

A síndrome de disautonomia pós-traumática representa uma afecção grave, frequentemente subestimada na assistência ao paciente com neurotrauma e que resulta em piora na recuperação funcional e elevação dos custos hospitalares por aumento do tempo de internação. Apresenta tratamento complexo e a abordagem em alguns casos representa um grande desafio para a equipe de assistência.

\section{Referências}

1. Andrade AF, Paiva WS, Amorim RL, Figueiredo EG, Rusafa Neto E, Teixeira MJ. [The pathophysiological mechanisms following traumatic brain injury]. Rev Assoc Med Bras. 2009;55(1):75-81.

2. Andrade AF, Paiva WS, Soares MS, De Amorim RL, Tavares WM, Teixeira MJ. Classification and management of mild head trauma. Int J Gen Med. 2011;4:175-9.

3. Kelly DF, Doberstein C, Becker DP. General principles of head injury management. In: Narajan RK, Wilberger JE, Povlishok JT, editors. Neurotrauma. New York: McGrawHill; 1996. p. 71-101.

4. Adekoya N, Majumder R. Fatal traumatic brain injury, West Virginia, 1989-1998. Public Health Rep. 2004;119(5):486-92. 
5. Cameron PA, Rainer TH, Mak P. Motor vehicle deaths in Hong Kong: opportunities for improvement. J Trauma. 2004;56(4):890-3.

6. Baguley IJ, Heriseanu RE, Gurka JA, Nordenbo A, Cameron ID. Gabapentin in the management of dysautonomia following severe traumatic brain injury: a case series. J Neurol Neurosurg Psychiatry. 2007;78(5):539-41.

7. Baguley IJ. The excitatory: inhibitory ratio model (EIR model): an integrative explanation of acute autonomic over activity syndromes. Med Hypotheses. 2008;70(1):26-35.

8. Blackman JA, Patrick PD, Buck ML, Rust RS Jr. Paroxysmal autonomic instability with dystonia after brain injury. Arch Neurol. 2004;61(3):321-8.

9. Baguley IJ, Nicholls JL, Felmingham KL, Crooks J, Gurka JA, Wade LD. Dysautonomia after traumatic brain injury: a forgotten syndrome? J Neurol Neurosurg Psychiatry. 1999;67(1):39-43.

10. Lv LQ, Hou LJ, Yu MK, Qi XQ, Chen HR, Chen JX, et al. Risk factors related to dysautonomia after severe traumatic brain injury. J Trauma. 2011;71(3):538-42.

11. Boeve BF, Wijdicks EF, Benarroch EE, Schmidt KD. Paroxysmal sympathetic storms ("diencephalic seizures") after severe diffuse axonal head injury. Mayo Clin Proc. 1998;73(2):148-52.

12. Penfield W, Jasper H. Autonomic seizures. In: Penfield W, Jasper $\mathrm{H}$. Epilepsy and the functional anatomy of the human brain. Boston: Little Brown; 1954. p. 412-37.

13. Baguley IJ, Heriseanu RE, Cameron ID, Nott MT, SlewaYounan S. A critical review of the pathophysiology of dysautonomia following traumatic brain injury. Neurocrit Care. 2008;8(2):293-300.

14. Rabinstein AA. Paroxysmal autonomic instability after brain injury. Arch Neurol. 2004;61(10):1625.

15. Russo RN, O'Flaherty S. Bromocriptine for the management of autonomic dysfunction after severe traumatic brain injury. J Paediatr Child Health. 2000;36(3):283-5.

16. Cuny E, Richer E, Castel JP. Dysautonomia syndrome in the acute recovery phase after traumatic brain injury: relief with intrathecal Baclofen therapy. Brain Inj. 2001;15(10):917-25.

17. Baguley IJ. Autonomic complications following central nervous system injury. Semin Neurol. 2008;28(5):716-25.

18. Baguley IJ, Cameron ID, Green AM, Slewa-Younan S, Marosszeky JE, Gurka JA. Pharmacological management of dysautonomia following traumatic brain injury. Brain Inj. 2004;18(5):409-17. PubMed PMID: 15195790.

19. Wijnen VJ, Heutink M, Van Boxtel GJ, Eilander HJ, De Gelder B. Autonomic reactivity to sensory stimulation is related to consciousness level after severe traumatic brain injury. Clin Neurophysiol. 2006;117(8):1794-807.

20. Wang VY, Manley G. Recognition of paroxysmal autonomic instability with dystonia (PAID) in a patient with traumatic brain injury. J Trauma. 2008;64(2):500-2.

Endereço para correspondência

Wellingson Silva Paiva

Rua Dr. Enéas de Carvalho Aguiar, 255

05403-010 - São Paulo, SP, Brasil

E-mail: wellingsonpaiva@yahoo.com.br 